\title{
1 MonaGO: a novel Gene Ontology enrichment analysis visualisation system
}

2

3 Ziyin Xin ${ }^{1,+}$, Yujun Cai ${ }^{1,3,+}$, Louis T. Dang ${ }^{2,4}$, Hannah M.S. Burke ${ }^{2,4}$, Jerico Revote ${ }^{5}$ Hieu T.

$4 \quad \operatorname{Nim}^{1,2,4,6, *}$, Yuan-Fang $\mathbf{L i}^{1,4, *}$, and Mirana Ramialison ${ }^{2,4,6, *}$

5

$6 \quad{ }^{1}$ Faculty of IT, Monash University, Clayton, VIC, Australia

$7 \quad{ }^{2}$ Australian Regenerative Medicine Institute, Monash University, Clayton, VIC, Australia

$8 \quad{ }^{3}$ Southeast University, Nanjing, China

$9 \quad{ }^{4}$ Systems Biology Institute, Clayton, VIC, Australia

$10{ }^{5}$ Monash eResearch Centre, Monash University, Melbourne, VIC, Australia

$11{ }^{6}$ Murdoch Children's Research Institute, Parkville, VIC, Australia

12

13

$14{ }^{*}$ Correspondence to:

15 hieu.nim@monash.edu

16 yuanfang.li@monash.edu

17 mirana.ramialison@monash.edu

18

$19{ }^{+}$these authors contributed equally to this work

Abstract

22

23 MonaGO is a novel web-based visualisation system that provides an intuitive, interactive and 24 responsive interface for performing gene ontology (GO) enrichment analysis and visualising the 
25 results. MonaGO combines dynamic clustering and interactive visualisation as well as

26 customisation options to assist biologists in obtaining meaningful representation of overrepresented

27 GO terms, producing simplified outputs in an unbiased manner. MonaGO supports gene lists as

28 well as GO terms as inputs. Visualisation results can be exported as high-resolution images or

29 restored in new sessions, allowing reproducibility of the analysis. An extensive comparison

30 between MonaGO and 11 state-of-the-art GO enrichment visualisation tools based on 9 features

31 revealed that MonaGO is the only platform that simultaneously allows interactive visualisation

32 within one single output page, directly accessible through a web browser with customisable display

33 options. In summary, MonaGO will facilitate the interpretation of GO analysis and will assist the

34 biologists into the representation of the results.

Keywords

37

gene ontology, GO enrichment, web services, interactive visualisation, semantic web

\section{Background}

42 Gene Ontology $(\mathrm{GO})^{1}$ is widely used in biomedical sciences to mine large-scale datasets. GO enrichment is one of the most popular post-omics analyses for datasets generated by genomics, transcriptomics, proteomics and metabolomics assays. A myriad of web-based tools or software packages are available to perform GO enrichments or classification, including the popular tools DAVID $^{2}$ and PANTHER ${ }^{3}$.

Inappropriate use of GO enrichment analyses can result in misleading targets and waste of 
50 predicted to be enriched above the statistical threshold, which of them should be displayed? Often 51 arbitrary decisions are made such as keeping only the "top 5 most-enriched" as a figure in 52 publications. In addition, the redundancy of GO terms due to its hierarchical nature makes 53 visualisation of enrichment results difficult, and often "representative terms" (e.g. "inflammation" 54 or "differentiation") are arbitrarily chosen to represent broadly-related GO categories. The 55 emerging field of visual analytics ${ }^{5}$ and its increasing use in biomedicine ${ }^{6}$ can bridge these 56 challenges by harnessing human expertise to navigate the dense information typically presented in 57 GO enrichment analyses, resulting in a meaningful representation of overrepresented GO terms.

We have developed MonaGO, a novel interactive online visualisation system for GO enrichment analysis results. MonaGO provides a coordinated interface that retains all information, yet remains intuitive, fluid, and easy to use for lay users. Therefore, MonaGO assists biologists in making informed decisions on which enriched terms should be displayed to allow a meaningful representation and interpretation of their datasets, without compromising on objectivity by arbitrarily choosing "representative terms".

Several tools exist that provide visualisation for GO enrichment analysis results ${ }^{7-9}$ but few permit on-the-fly exploration of GO terms clustering via chord diagram visualisation. The main advantages of MonaGO over these tools include (1) its intuitive and interactive interface and comprehensive interaction options, (2) the ability to manually or systematically cluster GO terms interactively, and (3) extensive input and output options.

\section{Implementation}

73

74 MonaGO utilises a client-server architecture and it comprises two main parts: (1) a frontend client 
75 receiving inputs from users and visualising the data, and (2) a backend server responsible for

76 processing data, querying database and producing data for visualisation. The client mainly in

77 JavaScript and server is built in Python.

The server consists of two Python modules. The first, server.py, utilizes Flask_1, a stable and scalable web application development framework. Specifically, when given a list of genes, this module sends a request to DAVID to obtain GO enrichment results. It also maintains a copy of the Gene Ontology hierarchy for visualising already enriched genes. Responses from DAVID are filtered and passed to the data-processing module. In addition, visualisation from a previously saved session can be restored by uploading a previously exported file, which already contains processed data. The sever.py module parses the file and sends it to client for visualisation directly. Redundant server nodes were implemented using the round-robin load balancing scheme to improve multi-user responsiveness.

The second module, dataprocess.py, performs data processing tasks, including calculating cluster similarity, creating hierarchical clusters, and reordering clusters for visualisation. Specifically, a

91 hierarchical clustering algorithm is employed to cluster GO terms into clusters according to one of 92 three options: the percentage of common genes between pairs of them (Jaccard similarity), the 93 Resnik similarity ${ }^{10}$ between gene pairs, and the SimRel similarity ${ }^{11}$ between gene pairs. Algorithm

941 (Figure 8) provides a more detailed description of this procedure. In this algorithm, the user inputs the type of similarity measurement and, if a semantic similarity is chosen, the way the similarity between clusters is determined (that is, whether to use the average, minimum or

97 maximum of the similarities between each combination of terms in the clusters).

99 Semantic similarities are calculated using the formulas described by Schlicker and Albrecht ${ }^{12}$. In 
100 order to evaluate these formulas, two databases are used; firstly, to count the number of annotations

101 of each GO term we use UniProtKB ${ }^{13}$ (updated 14/02/19). Secondly, to access the Gene Ontology

102 hierarchy we use go-basic.obo (accessed 05/03/19).

103

104 The client functions as a receiver and visualisation platform. MonaGO.js serves as the main

105 controller of functionalities. Dynamic and interactive graphics are generated using D3.js, a

106 JavaScript library allowing great control over final visualisation results. Through the visual

107 interface generated by the client, users can intuitively interact with the visualisation and download

108 high-resolution images from MonaGO, in PDF, PNG or SVG.

109

\section{Results and Discussion}

\section{MonaGO's interface allows a user-friendly interactive display of GO enrichment results}

113

114 MonaGO supports three different ways of data entry: (1) submitting a list of genes and using DAVID $^{2}$, one of the most widely used programs, to perform enrichment analysis in the

116 background, (2) submitting gene lists and associated, pre-selected enriched GO terms for 117 visualisation directly, and (3) importing a previously exported visualisation to restore it. MonaGO's 118 output options (Fig. 1) include high-resolution PNG or SVG images of a chord diagram (in the 119 main visualisation) and the ontology hierarchy of a GO term (in the details panel), as well as JSON 120 files that store the current state of the main chord diagram which can later be imported and restored in MonaGO.

123 The main visualisation interface (Fig. 1) comprises three main components: (A) the main 124 visualisation panel displays hierarchical clustering tree base on a chord diagram of enriched GO 
125 terms, (B) the "search box" panel allows browsing for specific terms or genes annotated by these

126 terms, and (C) a “details" panel displays further information on a specific GO term upon selection.

127

128 To provide biologists with a comprehensive representation of GO enrichment results, a chord

129 diagram (centre of Fig. 1) is employed as an intuitive and compact way to visualise clusters of GO

130 terms and similarity between them. Enriched GO terms are colour-coded based on their p-values

131 and their widths are proportional to the numbers of genes-of-interest contained in them.

132 The green edges parallel to the main chord diagram on the outside denote possible (hierarchical)

133 clusters, and the number on an edge represents the percentage of common genes-of-interest

134 between two nodes/clusters. The grey edges inside the chord diagram connect pairs of GO

135 term/clusters, and the presence of such an edge denotes the existence of common genes-of-interest

136 between them.

137

138 MonaGO helps reduce redundancy by hierarchically clustering similar GO terms in the main chord

139 diagram. In MonaGO, enriched terms are ordered in a way that similar GO terms are placed close to

140 each other. Users can choose between three distance metrics for the initial clustering GO terms:

141 percentage of overlapping genes or their semantic similarity (Resnik similarity ${ }^{10}$ and SimRel ${ }^{11}$ ).

142 When using Resnik and SimRel, users can choose between average, minimum or maximum options,

143 based on the semantic similarity between each combination of individual terms in the GO clusters.

144 Average takes the mean of all these similarities, and hence represents the distance between two

145 areas of the GO tree. Alternatively, minimum represents the distance between the farthest two nodes

146 of the clusters and maximum the closest two nodes. Hence minimum considers the Most

147 Informative Ancestor common to all GO terms in the clusters, whereas maximum considers the

148 Most Informative Ancestor of any two terms. 
150 Through this chord diagram display, users can easily cluster redundant GO terms as they wish,

151 thereby reducing the information overload. Clustering can be performed systematically or manually.

152 A slider at top left of the main component allows systematic clustering by controlling the threshold

153 of similarity score between terms or clusters. Alternatively, nodes/clusters in the chord diagram can

154 be manually collapsed and expanded.

155

156 Manual clustering functionality allows users to collapse GO terms/clusters by clicking edge-nodes.

157 For instance, as shown in Fig. 2A GO1 and GO2 share 11 common genes, which amounts to $100 \%$

158 of their total genes. If a user considers GO1 and GO2 to be highly redundant and wishes to group

159 them, the green nodes between them can be clicked and thus create a cluster (Fig. 2B).

160

161 High-resolution images of the chord diagram and the GO hierarchy of a selected GO term in the

162 details panel can be saved in three formats, PDF, SVG and PNG, by clicking the drop-down menu

163 button "Save image" at the top left of the main visualisation panel.

164 Moreover, a JSON file storing the current state of the main chord diagram and data of GO

165 enrichment results can be downloaded and later imported into MonaGO to restore the state of the

166 visualisation for subsequent analysis.

167

168 The details panel shows, for a node/cluster on the chord diagram or an edge inside the chord 169 diagram, additional information about it that is complementary to the main chord diagram (Fig. 1C).

170 For example, for an edge (highlighted with a green outline, Fig. 1A), the panel shows the number

171 and percentage of common genes between the two nodes (GO terms) it connects to, the list of these

172 genes and information of the semantic similarity between these terms (if chosen as similarity

173 measure) including a diagram of the GO hierarchy (Fig. 1C). The hierarchy diagram can be

174 expanded to full screen if needed, in order to view the tree more clearly. 
176 Finally, the search box provides a convenient alternative way of finding genes and their associated

177 GO terms by free-text search (Fig. 1). GO terms that correspond to a matched gene are listed in the 178 details panel as well as popped out in the chord diagram for easy identification (e.g. the highlighted 179 cluster "determination of liver left/right asymmetry in Fig. 1).

\section{MonaGO offers unique visualisation properties compared to existing tools}

To assess MonaGO's visualisation properties, we compared it to twelve well-known and highlycited GO analysis systems which offer a visualisation platform (Table 1) for GO enrichment analysis. Systems such as DAVID ${ }^{2}$ and PANTHER ${ }^{3}$, provide a large number of analytical services while not focussing on providing an interactive visualisation interface: tables or simple graphs are used to display enrichment results. Other systems such as REVIGO ${ }^{9}$, g:Profiler ${ }^{15}$, Gorilla ${ }^{16}$,

interface either based on existing results from GO enrichment analysis or performing GO enrichment from scratch through DAVID. Hence, where most of the systems accept GO terms user to (1) submit gene lists and perform the enrichment using DAVID ${ }^{7}$, (2) submitting GO terms with annotations directly, and (3) restoring previous visualisation results.

Node-link diagrams are widely used (e.g. BiNGO ${ }^{19}$, GOEAST ${ }^{20}$, Gorilla ${ }^{16}$, and WebGestalt ${ }^{17}$ ) when it comes to showing relationships between GO terms. However, the GO hierarchy or term-

197 term relationships are not easily shown in such an approach. To address this limitation, MonaGO

198 displays term-term similarity in a chord diagram while providing hierarchy and other information in 
200 interface. Some tools allow modification in the visualisation output (DAVID ${ }^{2}$, REVIGO ${ }^{9}$, and

201 WebGestalt ${ }^{17}$ ) by reloading the display after re-setting the parameters of interest (such as setting

202 threshold). Other tools (g:Profiler ${ }^{15}$, Gorilla ${ }^{16}$, GOplot ${ }^{18}$ ) only provide static interfaces/images.

203 However with MonaGO, changes in the visualisation parameters are simultaneously reflected on the

204 output display as the user modifies them.

205

MonaGO's interactive interface facilitates the reduction of enriched GO terms to display

207

MonaGO is one of a few GO visualisation tools that display the relationship between terms based on the number of common genes. To illustrate the advantages of MonaGO, we re-analysed our published datasets where we measured gene expression changes for three cells types (fibroblasts, neutrophils and keratinocytes) while reprogramming the cells into a pluripotent state ${ }^{21}$. In brief, genes sharing similar expression levels over five stages of reprogramming were clustered using cmeans fuzzy clustering. GO term enrichment for selected clusters was performed using DAVID ${ }^{7}$.

214 DAVID's default display output is a list of terms or cluster of terms (Figure 3Ai). In this test 215 dataset, several over-represented GO terms were found enriched. Due to the length of the list, it is 216 thus not uncommon that only the most statistically significant terms or terms relevant to the 217 biological question are retained, creating selection bias of GO terms (Figure 3Ai). In contrast, 218 MonaGO displays all over-represented terms (Figure 3Bi) in a single view, which can be further 219 systematically reduced into more generic clusters (Figure 3Bii), using the overlapping number of 220 genes as a threshold, knowledge of genes common to these clusters can be further capitalised to 221 unravel the molecular mechanisms driving these enriched biological processes. In DAVID, this 222 information is accessible through the cluster display mode (Figure 3Aii), where genes shared 223 between enriched GO terms within a cluster are listed as a static heatmap. In MonaGO, at any stage 224 during the clustering process, the genes shared between the clusters are accessible in the chord 
225 diagram (Figure 3Bii) which will assist in the interpretation of the data. For instance, in the test

226 dataset, the most significant term "immune response" has been clustered under "immune system

227 process", however genes in this category are also involved in other biological processes. For

228 example, out of 35 genes in the top category, six genes (Ccl5, Tac1,Cccr5, Ccr1, Clec5a and

229 Cd300c2) are also involved in 'cell-cell signaling'. MonaGO thereby allows the users to establish

230 functional links between terms that are otherwise just presented as disjoint items in a list. Using the

231 fibroblast dataset on $\mathrm{REVIGO}^{9}$, reduction of number of GO terms is effective and visualisation of

232 these similar GO terms is clear (Figure 3Ci), based on hierarchy level and p-value. Similar clusters

233 are retrieved through MonaGO (Figure 3Cii), however the inclusion of common genes to GO

234 clustering provides a unique perspective on the functional relationships between GO enriched

235 terms.

236 In conclusion, MonaGO's chord-diagram based interface allows an unbiased exploration of GO

237 clustering results. By supporting systematic clustering of GO terms and displaying the relationships

238 between the terms that are directly informed from the dataset, MonaGO produces meaningful

239 representation of overrepresented GO terms in an unbiased manner.

Clustering of GO terms by overlapping genes or semantic similarity simplifies the GO output and

reveals novel functional properties

244 MonaGO offers two distance similarity measurement options to cluster the enriched GO terms in

245 the chord diagram. We assessed the biological outcomes resulting from using Resnik semantic

246 similarity versus percentage of overlapping genes, using an in-house curated list of zebrafish

247 embryonic cardiac genes (Additional File 1). We used MonaGO to assess which biological

248 functions compose the developmental circuitry of the heart. 
250 Running MonaGO using "official gene symbol" as the identifier and "percentage of overlapping

251 genes' as the distance measurement allows to build a workable shortlist of biological functions that

252 are over-represented in this gene set. As an example, running the cardiac gene set in this mode

253 identified two different neighbouring terms 'central nervous system projection neuron

254 axonogenesis' and 'anterior/posterior axon guidance' sharing 100\% of overlapping genes (Figure

255 4A), hence suggesting that despite being described by different names, these two categories may

256 represent the same function. This is further confirmed by reperforming this test using 'Resnik

257 similarity (average)', where these two terms are still grouped into the same cluster (Figure 4B).

258 Investigation of the GO hierarchy shared between the terms, which is also a feature of MonaGO,

259 explains that their functional similarity pertains to 'axonogenesis' (Figure 5). Hence biologists can

260 be confident that grouping the terms into a single node is a valid operation which also helps reduce

261 information repetition.

262

263 Running the cardiac gene set with "Resnik similarity (average)" as similarity measure revealed that

264 some GO terms cluster together despite having no overlapping genes (Figure 6). Namely, the term

265 'liver development', 'thyroid gland development' and 'determination of liver left/right asymmetry'

266 form a cluster even though there is no grey edge linking the neighbours. Thus, clustering by

267 semantic similarity allowed us to identify two closely functionally related biological processes that

268 are recruited in the formation of the heart, despite the lack of overlap in the genes sets composing

269 these two processes.

270

271 Since this gene set is found to be active in the heart of zebrafish, we further interrogated the

272 functional link between liver and thyroid gland development (Figure 6) and heart development.

273 Neighbouring clusters in the chord diagram highlighted terms related to 'left/right asymmetry',

274 including 'determination of heart left/right asymmetry'. This suggests that heart, liver and thyroid 
275 gland development share common pathways during the determination of the left-right asymmetry

276 of these organs. This common ancestor link is confirmed by the GO hierarchy (Figure 7) and

277 supported by biological evidence as 'liver left/right asymmetry' and 'determination of heart

278 left/right asymmetry' show $20 \%$ of overlapping genes. Most importantly, the remaining genes that

279 belong to the liver clustered and that do not overlap with the heart cluster are of great interest for

280 the biologists. Indeed, clustering by semantic similarity allowed them to explore a novel hypothesis

281 that genes belonging the liver term are novel genes involved in the regulation of heart left-right

282 asymmetry.

283

284

\section{Conclusions}

286 MonaGO is a novel web-based visualisation with unique features enabling biologists with no 287 programming knowledge to interactive explore the GO clustering hierarchy to rapidly deduce 288 biological interpretations. To demonstrate the benefits of MonaGO using real-world problems from developmental biologists, our platform has shown novel biological insights that may have been overlooked using traditional non-interactive exploration of the GO hierarchy. Used in combination,

291 MonaGO's two distance measurements provide a framework to cluster terms with optimal 292 biological relevance and simplify the original input, even in the absence of previously known 293 functional relationships. As a result, MonaGO aims to provide a unique tool for biologists who are 294 interested in hands-on interaction with the gene lists and their semantic relationship to derive 295 biological interpretation.

\section{DECLARATIONS}


299 Ethics approval and consent to participate. No ethics approval and consent required for this

300 study.

301 Consent for publication. All authors provide consent for publication.

302 Competing interests. The authors declare no competing financial interests.

303 Funding. This work was supported by the Australian Research Council Discovery Project grants

304 DP140100077 to Y.-F.L., DP140101067 to M.R.; a National Health and Medical Research

305 Council/Heart Foundation Career Development Fellowship (1049980) and Sun foundation to M.R.,

306 the China Scholarship Council for Y.C. The Australian Regenerative Medicine Institute is

307 supported by grants from the State Government of Victoria and the Australian Government.

308 Authors' contributions. M.R. and Y.-F.L. conceived the research concept and wrote the paper,

309 Z.X. and Y.C. implemented the system and performed system comparison and analysis. L.D.

310 provided detailed feedback to the system. HMB implemented additional distance functions for the

311 clustering algorithm, improved the interactive visualisation, performed case studies, and revised the

312 manuscript. HTN provided data visualisation supervision and revised the manuscript. All authors

313 reviewed and approved the manuscript.

314 Availability of data and materials. All data generated or analysed during this study are included

315 in this published article and its supplementary information files, or directly available on the

316 MonaGO webpage. MonaGO is freely available at http://monago.erc.monash.edu/ with all major

317 browsers supported. The source code is available at https://github.com/liyuanfang/MonaGO.

318 Acknowledgments. We thank Dr. Cristina Keightley and members of the Monash Bioinformatics

319 Platform for their valuable feedback. We thank the Monash eResearch platform for their support 320 with the server. 
322

323 1. Ashburner M, Ball CA, Blake JA, Botstein D, Butler H, Cherry JM, et al. Gene Ontology:

324 tool for the unification of biology. Nature Genetics. 2000;25:25.

325 2. Sherman BT, Huang DW, Bryant D, Liu D, Lane HC, Kir J, et al. DAVID Bioinformatics

326 Resources: expanded annotation database and novel algorithms to better extract biology from large

327 gene lists. Nucleic Acids Research. 2007;35(suppl_2):W169-W75.

328 3. Muruganujan A, Casagrande JT, Poudel S, Mi H, Thomas PD. PANTHER version 10:

329 expanded protein families and functions, and analysis tools. Nucleic Acids Research.

330

331

332

333

334

335

336

337

338

339

340

341

342

343

344

345 2015;44(D1):D336-D42.

4. Fridrich A, Hazan Y, Moran Y. Too Many False Targets for MicroRNAs: Challenges and Pitfalls in Prediction of miRNA Targets and Their Gene Ontology in Model and Non-model Organisms. BioEssays. 2019;41(4):1800169.

5. Keim DA, Mansmann F, Schneidewind J, Thomas J, Ziegler H. Visual Analytics: Scope and Challenges. In: Simoff SJ, Böhlen MH, Mazeika A, editors. Visual Data Mining: Theory, Techniques and Tools for Visual Analytics. Berlin, Heidelberg: Springer Berlin Heidelberg; 2008. p. $76-90$.

6. Qu Z, Lau CW, Nguyen QV, Zhou Y, Catchpoole DR. Visual Analytics of Genomic and Cancer Data: A Systematic Review. Cancer Informatics. 2019;18:1176935119835546.

7. Huang DW, Sherman BT, Tan Q, Kir J, Liu D, Bryant D, et al. DAVID Bioinformatics Resources: expanded annotation database and novel algorithms to better extract biology from large gene lists. Nucleic Acids Research. 2007;35(suppl_2):W169-W75.
8. Mi H, Poudel S, Muruganujan A, Casagrande JT, Thomas PD. PANTHER version 10: expanded protein families and functions, and analysis tools. Nucleic Acids Research. 2015;44(D1):D336-D42. 
346 9. Supek F, Bošnjak M, Škunca N, Šmuc T. REVIGO Summarizes and Visualizes Long Lists

347 of Gene Ontology Terms. PLOS ONE. 2011;6(7):e21800.

348 10. Resnik P. Using information content to evaluate semantic similarity in a taxonomy.

349 Proceedings of the 14th international joint conference on Artificial intelligence - Volume 1;

350 Montreal, Quebec, Canada. 1625914: Morgan Kaufmann Publishers Inc.; 1995. p. 448-53.

351 11. Schlicker A, Rahnenführer J, Albrecht M, Lengauer T, Domingues FS. GOTax:

352 investigating biological processes and biochemical activities along the taxonomic tree. Genome 353 biology. 2007;8(3):R33-R.

354 12. Albrecht M, Schlicker A. FunSimMat: a comprehensive functional similarity database.

355 Nucleic Acids Research. 2007;36(suppl_1):D434-D9.

356 13. UniProt C. The Universal Protein Resource (UniProt) in 2010. Nucleic acids research.

357 2010;38(Database issue):D142-D8.

358 14. Bostock M, Ogievetsky V, Heer J. D ${ }^{3}$ Data-Driven Documents. IEEE Transactions on

359 Visualization and Computer Graphics. 2011;17(12):2301-9.

360 15. Peterson H, Hansen J, Reimand J, Kull M, Vilo J. g:Profiler-a web-based toolset for

361 functional profiling of gene lists from large-scale experiments. Nucleic Acids Research.

362 2007;35(suppl_2):W193-W200.

363 16. Eden E, Navon R, Steinfeld I, Lipson D, Yakhini Z. GOrilla: a tool for discovery and 364 visualization of enriched GO terms in ranked gene lists. BMC Bioinformatics. 2009;10(1):48.

365 17. Zhang B, Snoddy J, Kirov S. WebGestalt: an integrated system for exploring gene sets in 366 various biological contexts. Nucleic Acids Research. 2005;33(suppl_2):W741-W8.

367 18. Ricote M, Walter W, Sánchez-Cabo F. GOplot: an R package for visually combining 368 expression data with functional analysis. Bioinformatics. 2015;31(17):2912-4.

369 19. Heymans K, Kuiper M, Maere S. BiNGO: a Cytoscape plugin to assess overrepresentation 370 of Gene Ontology categories in Biological Networks. Bioinformatics. 2005;21(16):3448-9. 
371 20. Zheng Q, Wang X-J. GOEAST: a web-based software toolkit for Gene Ontology

372 enrichment analysis. Nucleic Acids Research. 2008;36(suppl_2):W358-W63.

373 21. Nefzger CM, Rossello FJ, Chen J, Liu X, Knaupp AS, Firas J, et al. Cell Type of Origin

374 Dictates the Route to Pluripotency. Cell Rep. 2017;21(10):2649-60.

375 22. Du Z, Zhou X, Ling Y, Zhang Z, Su Z. agriGO: a GO analysis toolkit for the agricultural

376 community. Nucleic Acids Research. 2010;38(supp1_2):W64-W70.

377 23. Carbon S, Ireland A, Mungall CJ, Shu S, Marshall B, Lewis S, et al. AmiGO: online access

378 to ontology and annotation data. Bioinformatics (Oxford, England). 2009;25(2):288-9.

379 24. Tripathi S, Pohl Marie O, Zhou Y, Rodriguez-Frandsen A, Wang G, Stein David A, et al.

380 Meta- and Orthogonal Integration of Influenza "OMICs" Data Defines a Role for UBR4 in Virus

381 Budding. Cell Host \& Microbe. 2015;18(6):723-35.

382

Figures, tables and additional files

384

385 Figure 1. The main visualisation interface of MonaGO consisting of three components: (A, left)

386 the main visualisation panel on the left that shows the chord diagram of GO terms that can be 387 hierarchically and dynamically clustered, (B, top right) search box, and (C, bottom right) the details 388 panel with dynamic GO hierarchy visualisation.

Figure 2. An example usage of the manual clustering feature of MonaGO: (A) the GO chord 391 diagram before clustering where GO1 and GO2 are to be merged and, (B) the GO chord diagram 392 after clustering.

393

394 Figure 3: Using MonaGO to study functions of genes involved reprogramming fibroblasts to a 395 pluripotent states. (A) List of clustered terms from GO enrichment of these genes using DAVID: 
396 (A.i) term clustering table; (A.ii) common genes display. (B) MonaGO clustering result of the same 397 gene sets used in (A), showing (B.i) clustering of the full set of terms; and (B.ii) manual clustering

398 from fibroblast gene cluster 4 in Nefzget et al 2017. (C) Visualisation of genes from 6 399 representative fibroblasts clusters by (C.i) REVIGO and (C.ii) MonaGO.

400

401 Figure 4: (A) Section of MonaGO's visualisation with set of cardiac genes from zebrafish and 402 overlapping genes as distance measurement. The GO terms 'central nervous projection neuron 403 axonogenesis' and 'anterior/posterior axon guidance' showing 100\% of overlapping genes, are 404 highlighted in yellow. (B) Same visualisation as (A) but using Resnik similarity as distance 405 measurement instead.

406

407 Figure 5: GO hierarchy between the terms 'central nervous system project neuron axonegenesis 408 and 'anterior/posterior axon guidance.' These have Resnik similarity of 3.638, with their Most 409 Informative Ancestor being 'axonogenesis.'

Figure 6: Section of MonaGO visualisation with set of cardiac genes from zebrafish and Resnik similarity as distance measurement. The GO terms 'liver development', 'determination of liver left/right asymmetry' and 'thyroid gland development' form a cluster of semantically similar terms 414 with no genes overlap. This cluster shares overlapping genes with 'determination of heart left/right 415 asymmetry' (highlighted in yellow).

417 Figure 7: GO hierarchy between the terms 'determination of heart left/right asymmetry' and 418 'determination of liver left/right asymmetry.' These have Resnik similarity of 4.229, with their Most Informative Ancestor being 'determination of left/right symmetry.' 
bioRxiv preprint doi: https://doi.org/10.1101/2020.09.27.316067; this version posted September 29, 2020. The copyright holder for this preprint (which was not certified by peer review) is the author/funder, who has granted bioRxiv a license to display the preprint in perpetuity. It is made available under aCC-BY-NC-ND 4.0 International license.

421 Figure 8: MonaGO's hierarchical clustering algorithm (Algorithm 1) to produce the dynamic chord 422 diagram.

423

424 Additional file 1: Curated list of embryonic cardiac genes in zebrafish

425 Additional_file_1.txt 
Table 1. Comparisons of MonaGO with existing GO analysis systems with visualisation capabilities.

\begin{tabular}{|c|c|c|c|c|c|c|c|c|c|c|}
\hline System & Interactive & $\begin{array}{l}\text { Visualisation } \\
\text { of enriched } \\
\text { GO terms }\end{array}$ & $\begin{array}{l}\text { Platform/ } \\
\text { Depen- } \\
\text { dency }\end{array}$ & $\begin{array}{l}\text { Download/ } \\
\text { Save data }\end{array}$ & $\begin{array}{l}\text { Find redun- } \\
\text { dant terms/ } \\
\text { select impor- } \\
\text { tant terms }\end{array}$ & $\begin{array}{l}\text { Show re- } \\
\text { lationship } \\
\text { between } \\
\text { GO terms }\end{array}$ & $\begin{array}{l}\text { Flexible } \\
\text { switching } \\
\text { between } \\
\text { pages }\end{array}$ & $\begin{array}{l}\text { Flexible } \\
\text { threshold } \\
\text { (p-value, } \\
\text { etc.) }\end{array}$ & Input list & Examples \\
\hline MonaGO & Yes & $\begin{array}{l}\text { Chord dia- } \\
\text { gram }\end{array}$ & Browser & Yes & Yes & Yes & Yes & Yes & $\begin{array}{l}\text { Genes, GO } \\
\text { terms, previ- } \\
\text { ous visualisa- } \\
\text { tion }\end{array}$ & Yes \\
\hline agriGO 22 & No & $\begin{array}{l}\text { Node-link } \\
\text { diagram }\end{array}$ & Browser & Yes & Yes & Yes & $\begin{array}{l}\text { No (visu- } \\
\text { alisation a } \\
\text { subsequent } \\
\text { step/page) }\end{array}$ & Yes & Genes, Probes & Yes \\
\hline $\mathrm{AmiGO}^{23}$ & No & $\begin{array}{l}\text { Node-link } \\
\text { diagram }\end{array}$ & Browser & Yes & Yes & Yes & No & Yes & Genes & No \\
\hline $\mathrm{BiNGO}^{1 y}$ & Yes & $\begin{array}{l}\text { Node-link } \\
\text { diagram }\end{array}$ & Cytoscape & Yes & Yes & Yes & Yes & Yes & $\begin{array}{l}\text { Graph or gene } \\
\text { list input }\end{array}$ & Yes \\
\hline $\mathrm{DAVID}^{2}$ & No & $\begin{array}{l}\text { Clustering, } \\
\text { map }\end{array}$ & $\begin{array}{l}\text { Browser, } \\
\text { Program- } \\
\text { matically } \\
\text { (Java, Perl, } \\
\text { Python) }\end{array}$ & Yes & Yes & Yes & $\begin{array}{l}\text { No (several } \\
\text { pages) }\end{array}$ & No & Genes & Yes \\
\hline GOEAST $^{20}$ & No & $\begin{array}{l}\text { Node-link } \\
\text { diagram }\end{array}$ & Browser & Yes & Yes & Yes & Yes & Yes & Probe set ID & No \\
\hline GOplot $^{18}$ & No & $\begin{array}{l}\text { Circleplot, } \\
\text { Chord plot }\end{array}$ & $\mathrm{R}$ & Yes & Yes & Yes & No & Yes & $\begin{array}{ll}\text { GO terms } \\
\text { only }\end{array}$ & Yes \\
\hline Gorilla $^{16}$ & No & $\begin{array}{l}\text { Node-link } \\
\text { diagram }\end{array}$ & Browser & Yes & $\begin{array}{ll}\text { No } & \text { (ab- } \\
\text { stract } & \text { and }\end{array}$ & Yes & No & Yes & $\begin{array}{l}\text { Genes, Pro- } \\
\text { teins }\end{array}$ & Yes \\
\hline
\end{tabular}




\begin{tabular}{|c|c|c|c|c|c|c|c|c|c|c|}
\hline g:Profiler ${ }^{15}$ & No & $\begin{array}{l}\text { with high- } \\
\text { lights } \\
\text { Bar chart, } \\
\text { Word cloud }\end{array}$ & Browser & Yes & $\begin{array}{l}\text { redundant } \\
\text { hierarchy) } \\
\text { Yes (by rank- } \\
\text { ing) }\end{array}$ & $\begin{array}{l}\text { No (matrix } \\
\text { denotes } \\
\text { common } \\
\text { genes be- } \\
\text { tween GO } \\
\text { terms) }\end{array}$ & No & Yes & $\begin{array}{l}\text { Genes, Pro- } \\
\text { teins, Probes }\end{array}$ & Yes \\
\hline Metascape $^{24}$ & No & $\begin{array}{l}\text { Node-link } \\
\text { diagram, } \\
\text { Bar chart }\end{array}$ & $\begin{array}{l}\text { Browser, } \\
\text { Cytoscape }\end{array}$ & Yes & Yes & Yes & Yes & No & $\begin{array}{l}\text { RetSeq, } \\
\text { Ensembl, } \\
\text { Uniprot, } \\
\text { UCSC }\end{array}$ & Yes \\
\hline PANTHER $^{3}$ & Yes & Pie chart & Browser & Yes & Yes & Yes & $\begin{array}{l}\text { No (several } \\
\text { pages) }\end{array}$ & No & $\begin{array}{l}\text { Genes, Pro- } \\
\text { teins }\end{array}$ & $\begin{array}{l}\text { Yes (only } \\
\text { format, no } \\
\text { complete } \\
\text { examples) }\end{array}$ \\
\hline $\mathrm{REVIGO}^{9}$ & Yes & $\begin{array}{l}\text { Node link } \\
\text { diagram, } \\
\text { Tree map, } \\
\text { Word cloud, } \\
\text { Scatterplot }\end{array}$ & Browser & Yes & Yes & Yes & Yes & Yes & GO terms & Yes \\
\hline WebGestalt ${ }^{1 /}$ & Yes & $\begin{array}{l}\text { Node link } \\
\text { diagram }\end{array}$ & Cytoscape & Yes & Yes & $\begin{array}{l}\text { Yes (but no } \\
\text { interactive } \\
\text { details } \\
\text { about com- } \\
\text { mon gene } \\
\text { lists) }\end{array}$ & Yes & Yes & Genes & Yes \\
\hline
\end{tabular}




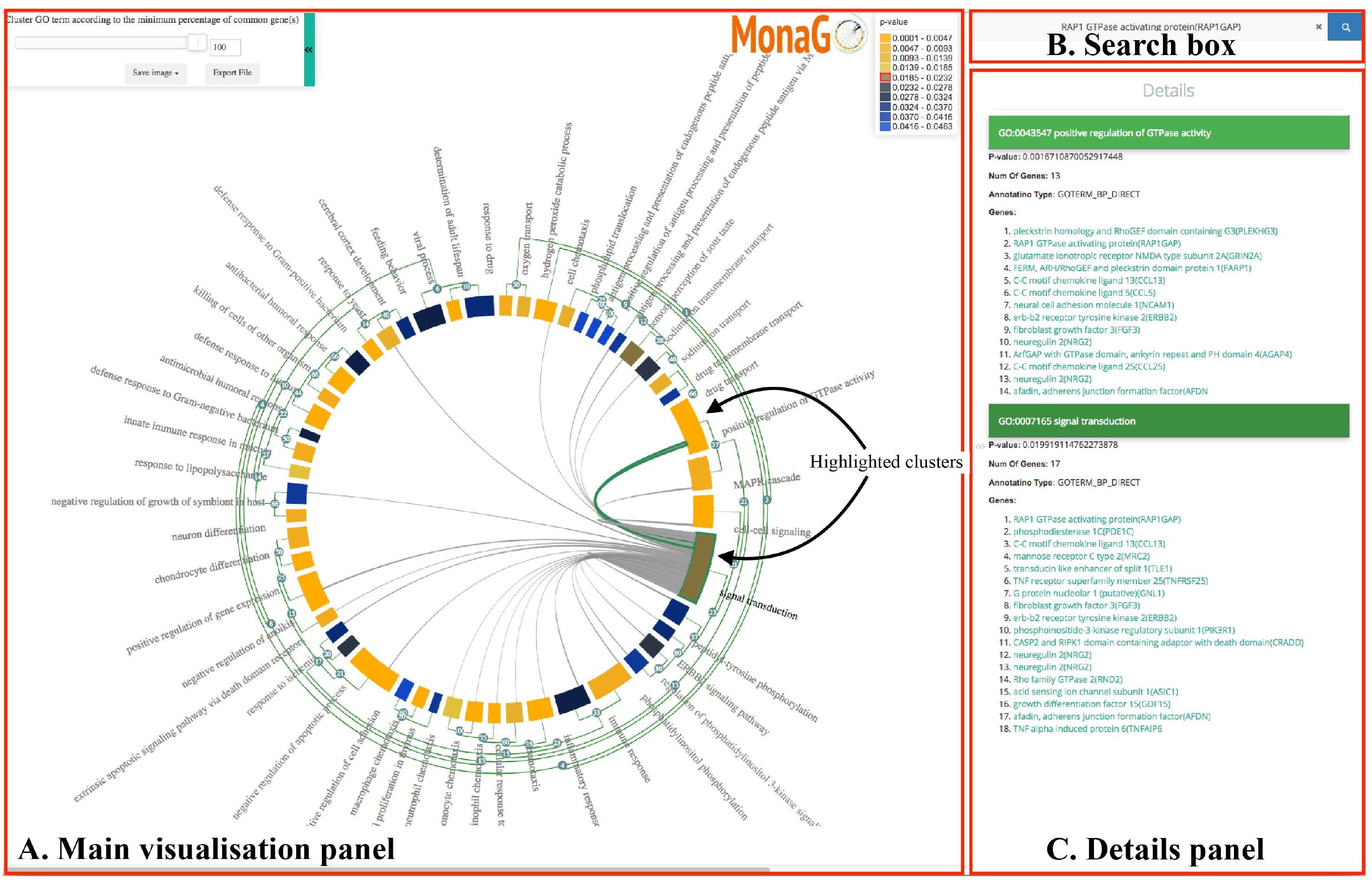




$$
Q Q
$$


ii.

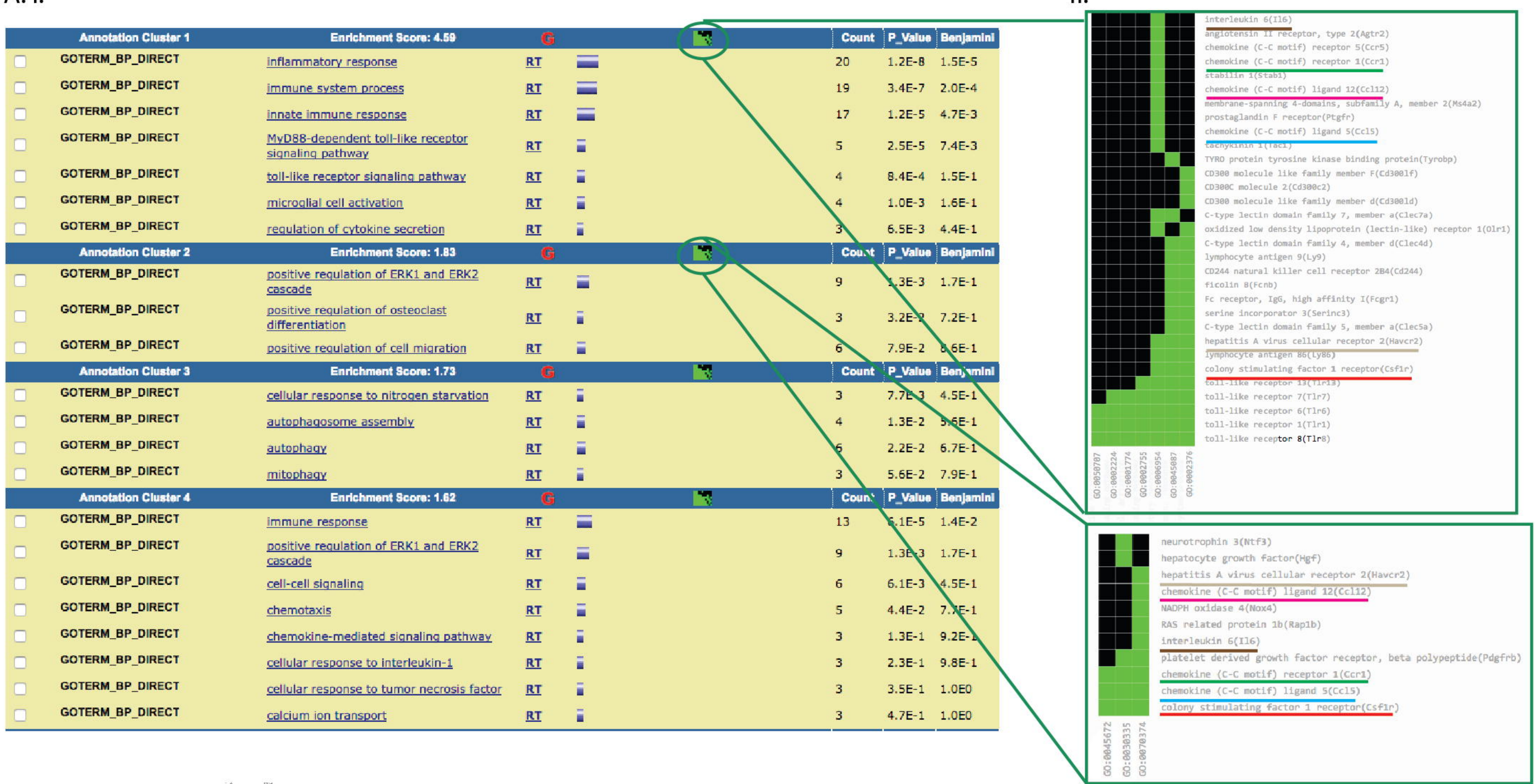

B.i.

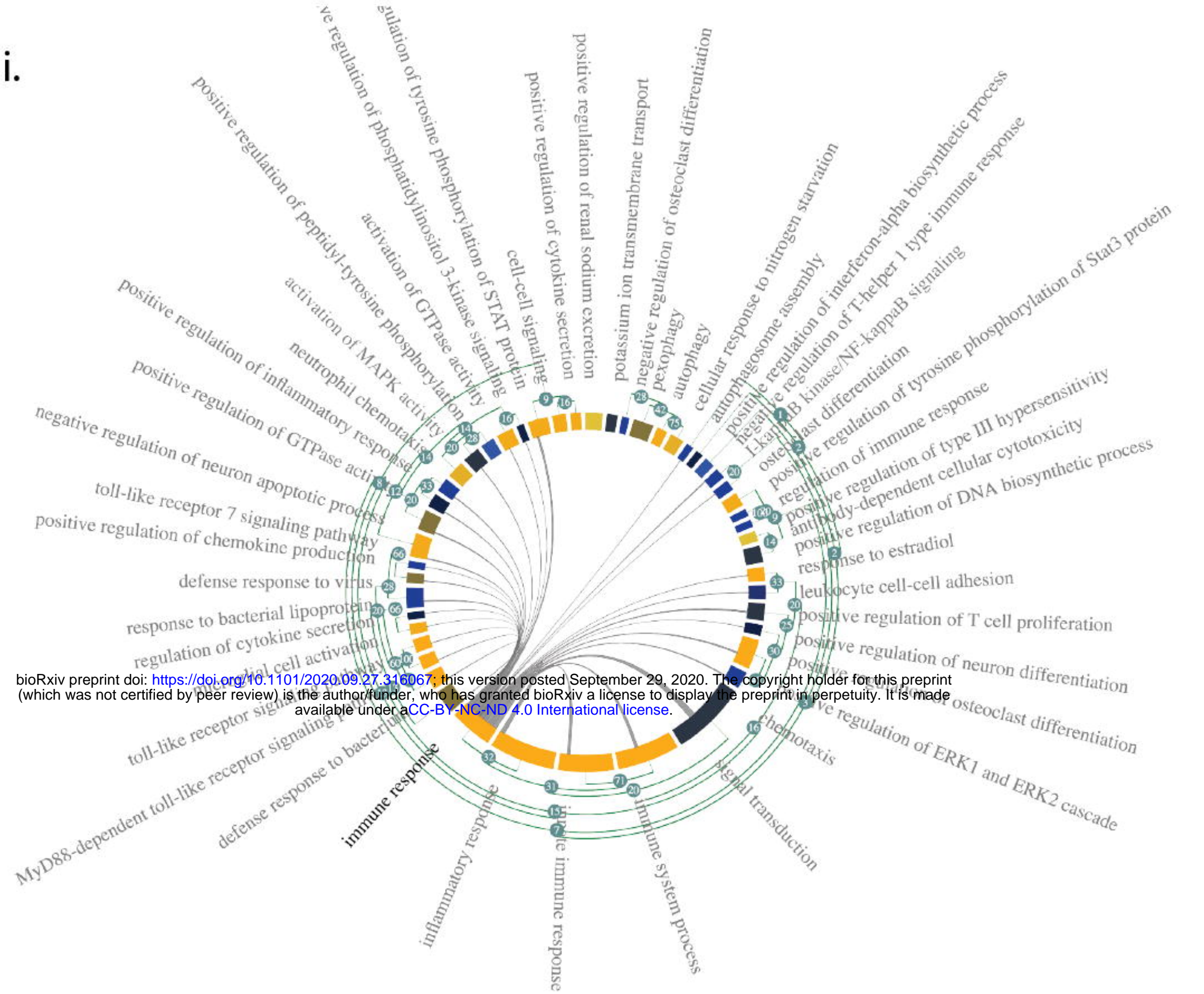

C.i.

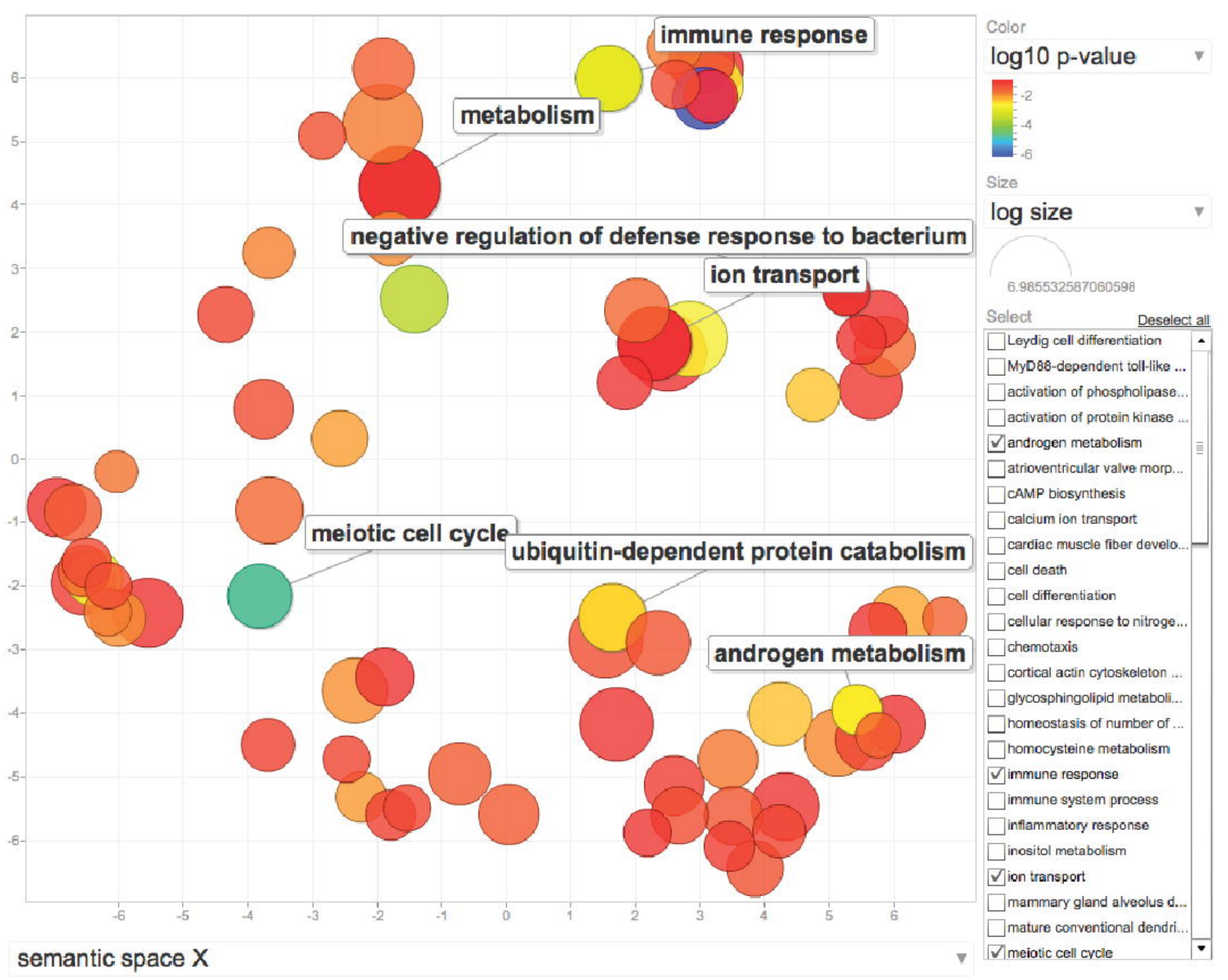

ii.

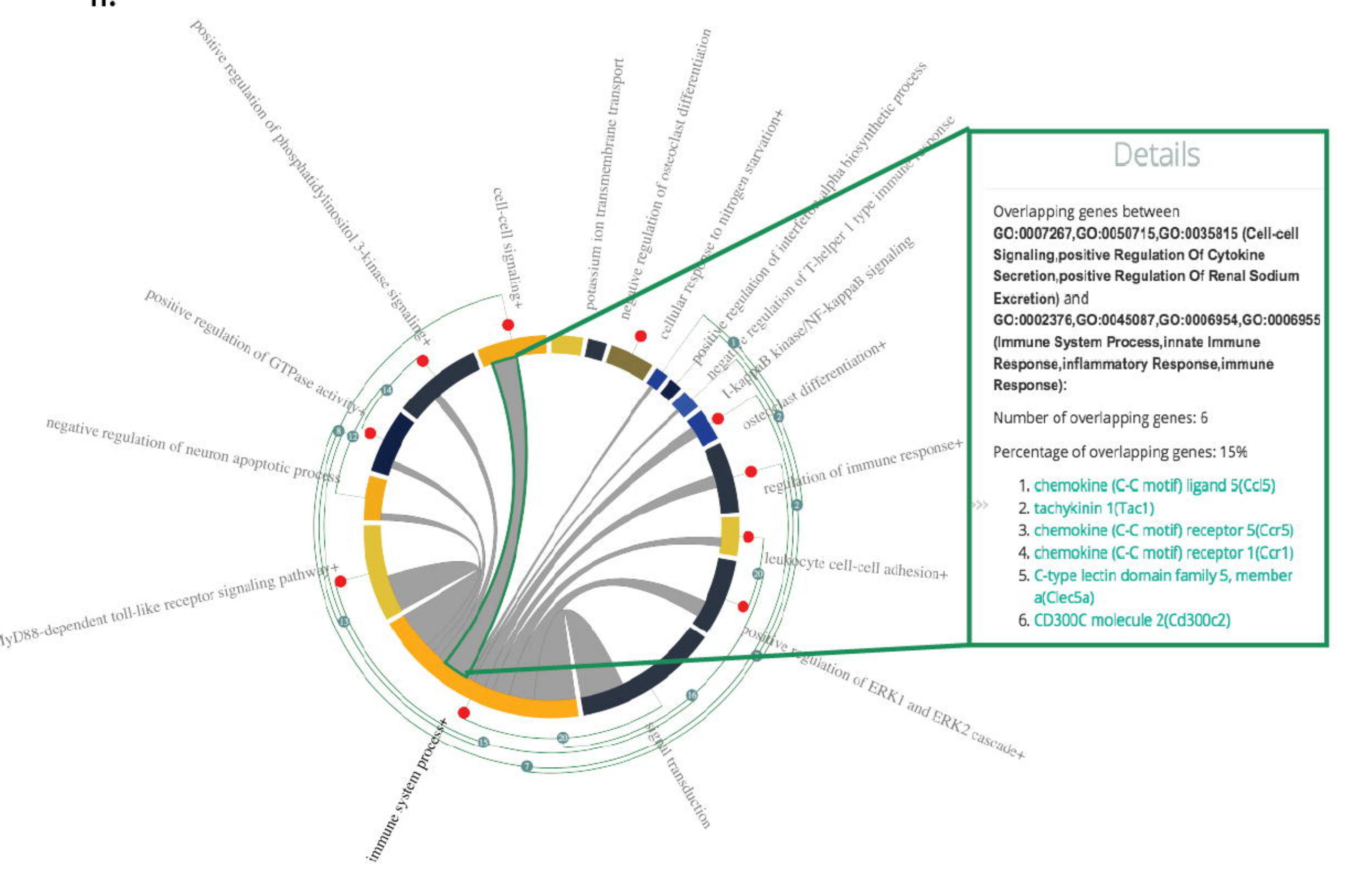

ii.

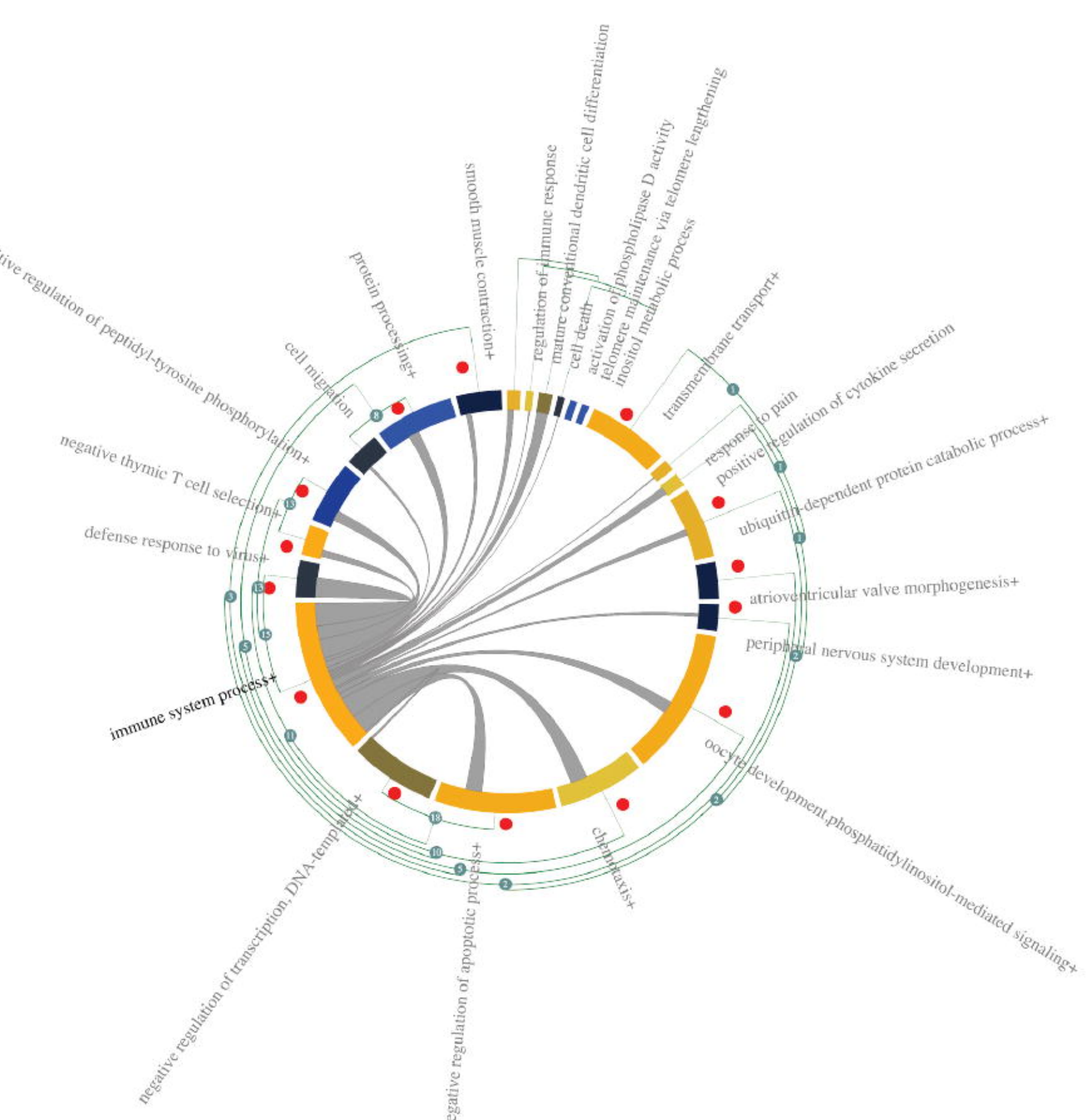


A

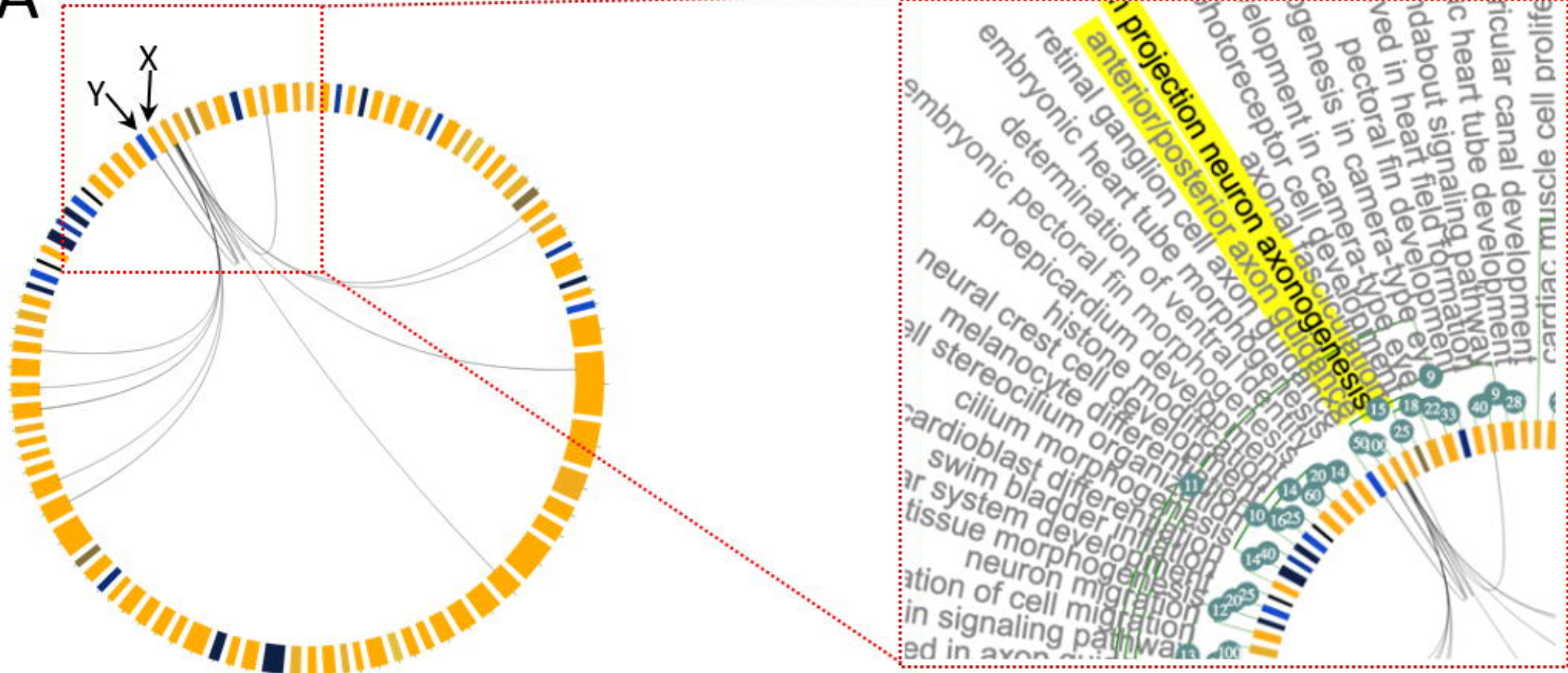

$\mathrm{X}$ : central nervous system projection neuron axonogenesis

$\mathrm{Y}$ : anterior posterior axon guidance

B

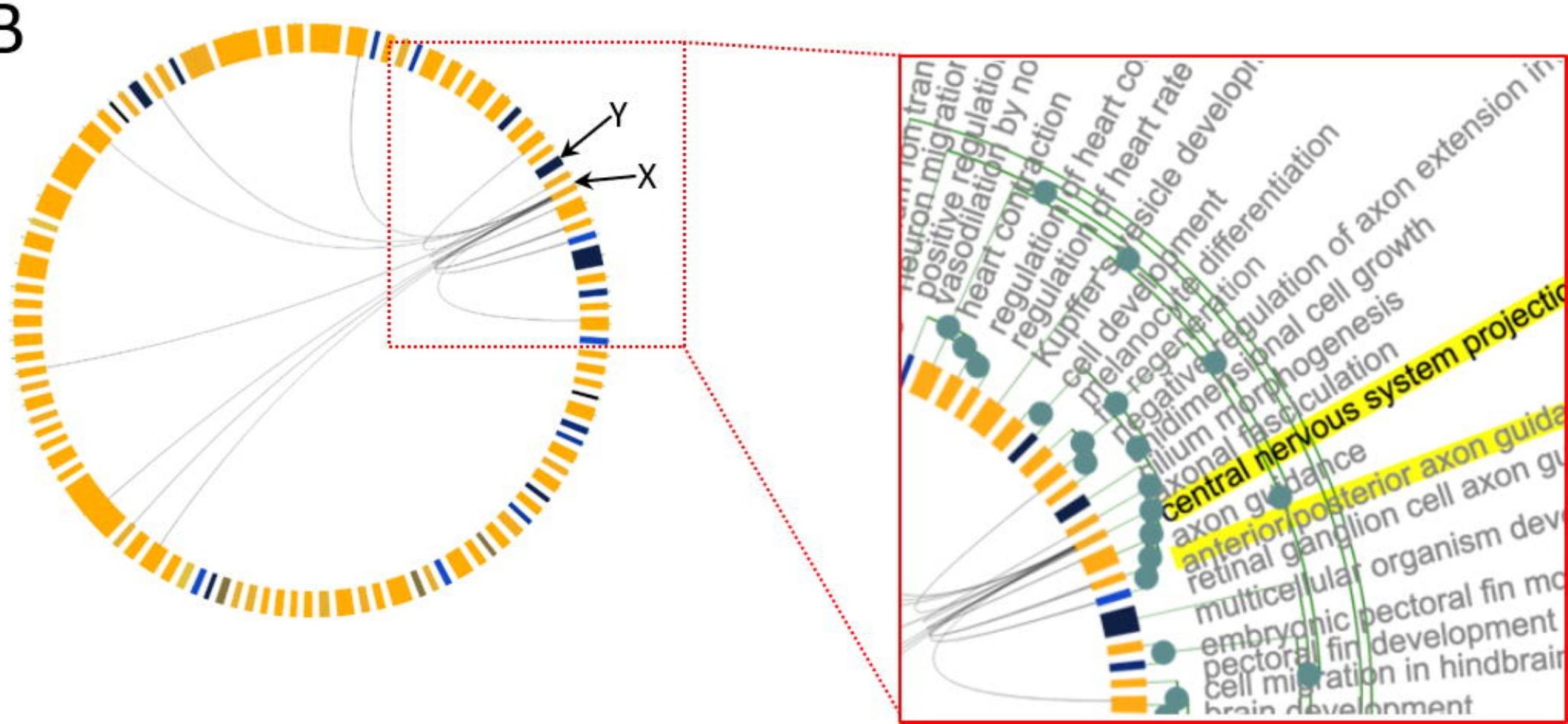




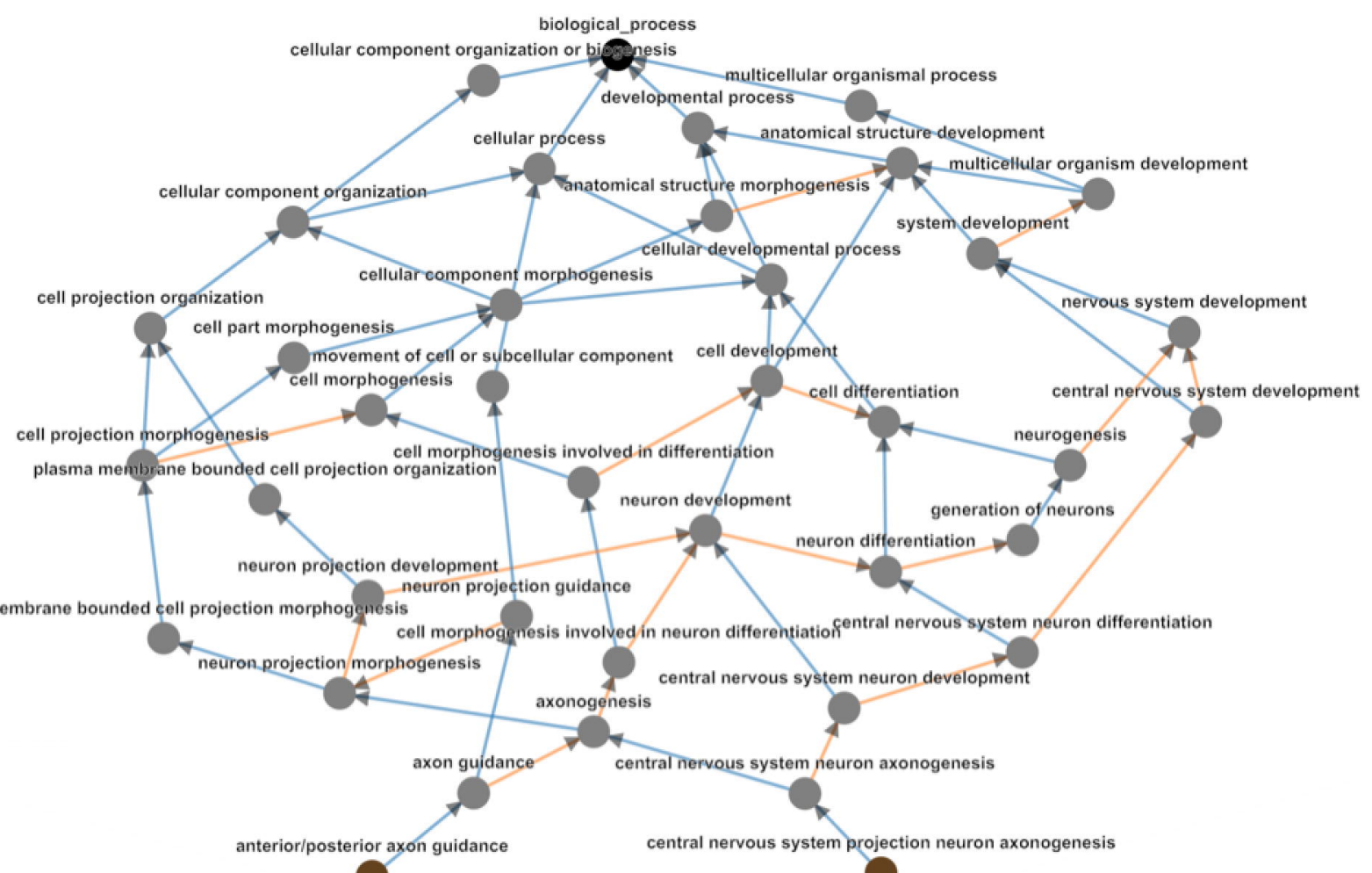
plasma membrane 


\section{biological_process}

is a

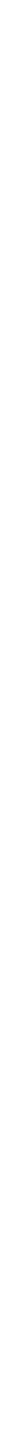


Algorithm 1 The hierarchical clustering algorithm.

Input: $M=\left\{G O_{1}, G O_{2}, \ldots, G O_{n}\right\}$ : a set of GO terms (initial clusters)

Output: $M$ : the updated new set of hierarchically-clustered GO terms

1: procedure CLUSTER $(M)$

2: $\quad$ while $|M|>1$ do

3: $\quad\left(G O_{i}^{*}, G O_{j}^{*}\right) \leftarrow \operatorname{argmax}_{1 \leq i<j \leq|M|} \operatorname{sIM}\left(G O_{i}, G O_{j}\right)$

$C l^{p} \leftarrow \operatorname{merge}\left(G O_{i}^{*}, G O_{j}^{*}\right)$

$M \leftarrow M \cup\left\{C l^{p}\right\}$

$M \leftarrow M \backslash\left(G O_{i}^{*}, G O_{j}^{*}\right)$

$\triangleright$ the number of clusters is greater than 1

$\triangleright$ pick two clusters with highest similarity

$\triangleright$ merge into the new parent cluster $C l^{p}$

$\triangleright$ add the new cluster $C l^{p}$ into the set

re-calculate similarity values for all pairs of top-level clusters

7 :

8:

9: function $\operatorname{sim}\left(C_{i}, C_{j}\right)$

10: $\quad$ if similarity measurement is number of overlapping genes then

11:

12:

else

return $\frac{\left|\operatorname{Genes}\left(C_{i}\right) \cap \operatorname{GenES}\left(C_{j}\right)\right|}{\left|\operatorname{GENES}\left(C_{i}\right) \cup \operatorname{GeNES}\left(C_{j}\right)\right|} * 100$

$\triangleright$ minimum percentage of overlapping genes

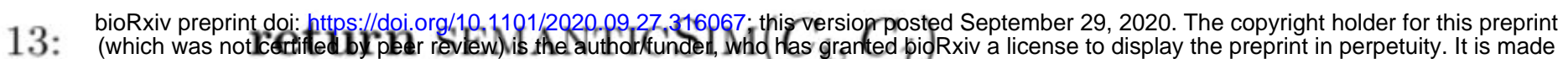

14: function SemanticSim $\left(C_{i}, C_{j}\right)$

15: $\quad$ termSims $\leftarrow$ []

16: $\quad$ for $t_{1}$ in $C_{i}$ do

$\triangleright$ initiate list to hold similarities between individual GO terms

17:

for $t_{2}$ in $C_{j}$ do

$\triangleright$ iterate through GO terms in clusters

18:

$M I A \leftarrow \operatorname{argmax}_{t_{a} \in \operatorname{Ancestors}\left(t_{1}\right) \cap \operatorname{ancestors}\left(t_{2}\right)} \operatorname{IC}\left(t_{a}\right)$

$\triangleright$ find Most Informative Ancestor

common to the two terms

19:

20:

21:

22:

23:

24:

25:

26:

27 :

28:

29:

30 :

31:

32

33

\section{4}

35

36

switch similarity measurement do

case Resnik

$\operatorname{sim} \leftarrow \mathrm{IC}(M I A)$

case SimRel

$$
\operatorname{sim} \leftarrow \frac{2 \log (\mathrm{P}(M I A))}{\log \left(\mathrm{P}\left(t_{1}\right)\right)+\log \left(\mathrm{P}\left(t_{2}\right)\right)}
$$

termSims $\leftarrow$ termSims $\cup$ sim

switch cluster comparison do

case average

return ave termSims

case minimum

return min termSims

case maximum

return max termSims

function $\mathrm{IC}($ term $)$

3: $\quad$ return $-\log (\mathrm{P}($ term $)$

4: function $\mathrm{P}($ term $)$

35: $\quad$ return $\frac{\text { FREQ }(\text { term })}{\operatorname{FREQ}(\operatorname{ROOT}(\text { term }))}$

36: function FREQ(term)

37: $\quad$ return ANNOT $($ term $)+\sum_{c \in \operatorname{CHLDREN}(\text { term })} \operatorname{FREQ}(c) \quad \triangleright$ recursive function to find frequency of term

When calculating the percentage of common genes in the function sim, genes $(\mathrm{Ci})$ returns the list of genes of the given cluster Ci.

When calculating the MIA of two GO terms in the function semanticSim, ancestors(term) gives a list of all ancestors of a GO term based on the GO hierarchy.

For calculating the probability of a GO term in the function p, root(term) returns the root node of the GO term, based on the GO hierarchy.

For calculating the frequency of a GO term in the function freq, annot(term) returns the number of annotations that term has in the UNIPROT database. The function children(term) gives a list of all children of a GO term based on the GO hierarchy. 